\title{
Synergism of INS and PDR in Self-Contained Pedestrian Tracking With a Miniature Sensor Module
}

\author{
Chengliang Huang, Zaiyi Liao, and Lian Zhao
}

\begin{abstract}
This paper presents a sensor-based pedestrian tracking technology that does not rely on any infrastructure. The information about human walking is monitored by a sensor module composed of accelerometers, gyroscopes and magnetometers. The acquired information is used by an algorithm proposed in this paper to accurately compute the position of a pedestrian. Through the application of human kinetics, the algorithm integrates two traditional technologies: strap-down inertial navigation and pedestrian dead-reckoning. Based on the algorithm, this paper presents several methods to improve the accuracy of pedestrian tracking through reducing the integral drift which is the main cause of errors in inertial navigation. These methods have been carefully investigated through theoretical study, simulation and field experiment. The results indicate accurate tracking is achievable through the application of both the proposed algorithm and methods. Evidently, it is feasible to develop self-contained pedestrian tracking system using inertial/magnetic sensors, eliminating the need for complicated and normally expensive infrastructure that most existing tracking systems rely on.
\end{abstract}

Index Terms-Human walking, inertial navigation system (INS), integral drift, pedestrian dead reckoning (PDR), pedestrian tracking, sensor module.

\section{NOMENCLATURE}

$\begin{array}{ll}\alpha & x \text { axis tilt. } \\ \beta & y \text { axis tilt. } \\ \psi & \text { Euler angle }- \text { yaw. } \\ \theta & \text { Euler angle }- \text { pitch. } \\ \phi & \text { Euler angle }- \text { roll. } \\ \omega & \text { Angular velocity. } \\ \varpi & \text { Euclidian of Angular velocity. } \\ m & \text { Magnetic field strength. } \\ a & \text { Acceleration. } \\ v & \text { Linear velocity. } \\ s & \text { Displacement. }\end{array}$

Manuscript received November 29, 2009; accepted February 12, 2010. Date of current version May 26, 2010. This work was supported in part by Ryerson Interdisciplinary Research Fund and by the National Sciences and Engineering Research Council of Canada (NSERC) research projects 313375-07 and 293237-04. The associate editor coordinating the review of this paper and approving it for publication was Prof. Giorgio Sberveglieri.

The authors are with the Electrical and Computer Engineering Department, Ryerson University, Toronto, ON, Canada, M5B 2K3 (e-mail: c28huang@ ryerson.ca; zliao@ryerson.ca; lzhao@ee.ryerson.ca).

Color versions of one or more of the figures in this paper are available online at http://ieeexplore.ieee.org.

Digital Object Identifier 10.1109/JSEN.2010.2044238 $t$ time.

D Magnetic declination.

\section{INTRODUCTION}

N OWADAYS, precise pedestrian tracking remains an open research problem. The accuracy of global positioning system (GPS) is degraded in thick forest, urban area with high buildings, and indoors. The deployment of most existing positioning systems based on infrastructure, such as infra-red, ultrasound, narrowband radio, wireless fidelity (WIFI) signal strength, ultra wide band (UWB), and vision [1], [2], is often difficult, if not impossible. For instance, the deployment and calibration of UWB beacon are too time-consuming for typical applications [3]. In principle, sensor nodes could be deployed to form an ad-hoc network but the accuracy is likely to be unsatisfactory with a limited number of arbitrarily-deployed nodes. Positioning with robotics, where inertial sensors, laser range-finders and computer vision are used, is not suitable for first respondent users such as a fire fighter.

Recent development of microelectromechanical systems (MEMS) allows for integrating multiple sensors, including orthogonal gyroscopes and accelerometers in a compact inertial sensor module. The technology is becoming cheaper and thus affordable for more applications. All the information needed for the detection of human movement is embedded in the signals generated by gyroscopes and accelerometers. This fact is employed in extensive research on pedestrian dead reckoning (PDR) and its applications [4]-[10]. The error of the step length calculated from accelerations measured on the waist, torso, or head can be as low as $3 \%$ to $10 \%$ [6], [7] but grows quickly if the user walks unsteadily or on slopes. This is because in PDR basically a pedestrian is tracked through summing up all the walking steps. The lengths and courses of the steps are assumed to satisfy certain statistic models, such as normal distribution [9].

On the other hand, pedestrian tracking can be treated as an application of the inertial navigation technology. Limited by the size and weight, the gimbaled inertial navigation system is not suitable for applications like pedestrian tracking. Instead, the strap-down inertial navigation system (INS) becomes a good candidate for the applications that require mobility. In a strapdown INS, the orientation of a sensor module is tracked by integrating the angular velocity signals, which are in turn used to calculate the acceleration components in the global coordinate system (GCS). After the acceleration of gravity is subtracted, the remaining acceleration is then double integrated over time to determine the displacement of the sensor module relative to a known starting point [11], [12]. However, cheap MEMS 
sensors are susceptible to drift errors. Small errors perturbing the gyroscope signals can cause growing angular errors in the tracked orientation. Other than the errors perturbing the acceleration signals, these angular errors can result in erroneous projection of gravity onto the horizontal axes in GCS. Consequently, the falsely projected acceleration of gravity is double integrated over time to yield substantial error of calculated displacement. The displacement drift incurred so may exceed $100 \mathrm{~m}$ after 1 min of integration [13]. Until recently, it was thought that pedestrian tracking with data from MEMS sensors was not possible due to the quadratic growth of errors caused by drift during double integration [13].

In order to realize a practical self-contained, infrastructurefree pedestrian tracking system, we propose a solution through the integration of both PDR and INS technologies and application of human kinetics. This integration is referred to as the synergism of INS and PDR in this paper. The positioning is obtained through tracking the length and orientation of each stride, just as what PDR does. The length and orientation of each stride are measured using the INS technology. Within a stride, the measurement is more accurate than the estimate using PDR technology, which is based on statistical estimation and does not directly measure the length and orientation of each stride. This paper will present the realization of the synergism of INS and PDR. It will also be explained how to solve the problems in tracking a person walking in different paths, using an off-theshelf wearable inertial/magnetic sensor module. Through experiments, we find that we are able to track a person with an accuracy of about $2 \%$, without need for the user to provide any explicit initialization information.

The remaining of this paper is organized as follows. Section II briefly describes the application of INS technology in the targeted systems with a focus on algorithms including coordinate transformation, dynamic orientation, and position tracking. Section III introduces algorithms for improving accuracy of tracking through merging PDR concept into INS technology. Novel methods are proposed while some existing methods are adopted. The prototyping of the proposed methodology is detailed in Section IV. The results of these experiments are demonstrated to evaluate the performance of the proposed algorithm and methods. Finally, Section V provides a conclusion to the latest research achievements and discusses further work to be conducted in the near future.

\section{INERTIAL TRACKING}

This section concerns with how a person is tracked with inertial sensors. Within a well defined coordinate transformation, detailed description is given on how dynamic orientation is estimated based on the signals from the sensors. A rotation matrix that relates the local coordinate system (LCS) to the GCS is estimated and updated based on the angular velocity outputs from the gyroscopes. Then, the acceleration in the LCS is transformed into acceleration in the GCS with this matrix. After deduction of the acceleration of gravity, the resulting acceleration is double integrated over time to yield a displacement in the GCS.

\section{A. Coordinate Systems and Transformation}

Fig. 1 shows the Cartesian coordinate system used in this paper. Axes $x^{w}, y^{w}$, and $z^{w}$, respectively, represent north, east,
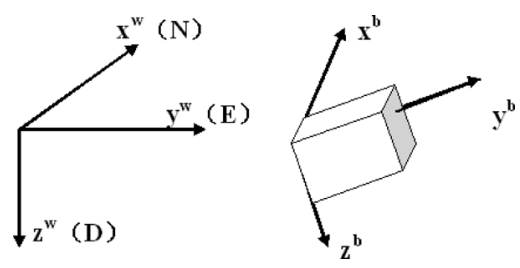

Fig. 1. Global coordinate system and the local coordinate system.

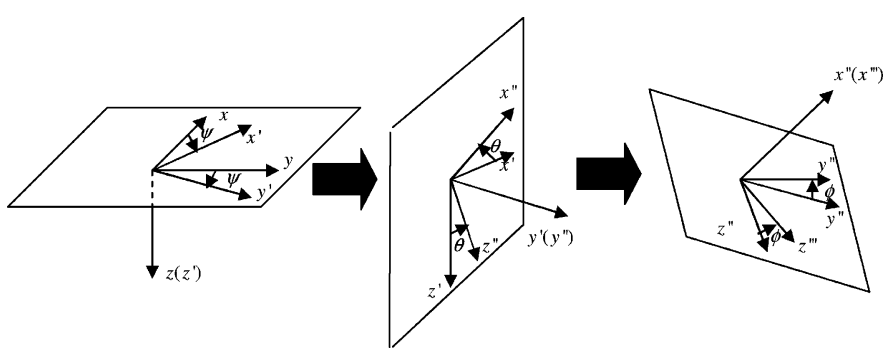

Fig. 2. Three sequential rotations from the GCS to LCS.

and down in GCS fixed to the surface of earth. The orientation of the sensor module will be estimated in this coordinate system. Axes $x^{b}, y^{b}$ and $z^{b}$ represent the orthogonal axes of the LCS, which is fixed to the sensor module.

In order to describe the components of a vector of arbitrary orientation in GCS with respect to the LCS, one of several rotation representations must be used. Most frequently used representations include direction cosines, Euler angles and quaternion [12]. In this paper, we use the direction cosines representation to develop algorithms to track the orientation of a sensor module.

Mathematically, the direct cosine matrix (DCM) is accomplished by transformation of three sequential rotations from the axes in the GCS, as illustrated in Fig. 2. These rotations continue as $\psi$ rotation about axis $z\left(z^{\prime}\right) ; \theta$ rotation about axis $y^{\prime}\left(y^{\prime \prime}\right)$, resulting from the first rotation; and, finally, $\phi$ rotation about axis $x^{\prime \prime}\left(x^{\prime \prime \prime}\right)$, resulting from the second rotation. These rotated angles are called yaw $(\psi)$, pitch $(\theta)$ and roll $(\phi)$, respectively. They are a specific kind of Euler angles. These rotations are denoted as $\mathbf{R}_{1}(\psi, z), \mathbf{R}_{2}(\theta, y)$, and $\mathbf{R}_{3}(\phi, x)$ respectively. The DCM representing transformation from the LCS to the GCS is obtained through transposing to $\mathbf{R}_{1}(\psi, z) \mathbf{R}_{2}(\theta, y) \mathbf{R}_{3}(\phi, x)$, resulting

$$
\mathbf{R}=\left(\begin{array}{ccc}
c \theta c \psi & -c \phi s \psi+s \phi s \theta c \psi & s \phi s \psi+c \phi s \theta c \psi \\
c \theta s \psi & c \phi c \psi+s \phi s \theta s \psi & -s \phi c \psi+c \phi s \theta s \psi \\
-s \theta & s \phi c \theta & c \phi c \theta
\end{array}\right)
$$

where $c$ stands for cos and $s$ stands for $\sin$.

\section{B. Dynamic Orientation Tracking}

The dynamic orientation, or the orientation of a moving object, is tracked by integrating the angular velocity vector $\omega(t)=$ $\left[\omega_{x}(t), \omega_{y}(t), \omega_{z}(t)\right]^{T}$, obtained from gyroscope.

In a short sampling period $\delta t$, let $\omega=\left[\omega_{x}, \omega_{y}, \omega_{z}\right]^{T}$ be the corresponding angular velocity sample, and $\delta \Psi=[\delta \psi, \delta \theta, \delta \phi]^{T}$ be small rotated angle vector the LCS has about its $z, y$ and $x$ axes, respectively. Hence, $\delta \Psi=\boldsymbol{\omega} \delta t$. When $\delta t$ is small, $\delta \phi, \delta \theta$ 
and $\delta \psi$ are small too. Using approximations and ignoring the products of angles in (1), the rotation matrix for this period is

$$
\mathbf{C}(t)=\left(\begin{array}{ccc}
1 & -\delta \psi & \delta \theta \\
\delta \psi & 1 & -\delta \phi \\
-\delta \theta & \delta \phi & 1
\end{array}\right)=\mathbf{I}+\boldsymbol{\Omega} \delta t
$$

where

$$
\boldsymbol{\Omega}=\left(\begin{array}{ccc}
0 & -\omega z & \omega y \\
\omega z & 0 & -\omega x \\
-\omega y & \omega x & 0
\end{array}\right)
$$

If the DCMs are $\mathbf{R}(t)$ and $\mathbf{R}(t+\delta t)$ at time $t$ and $t+\delta t$, respectively, $\mathbf{C}(t)$ is the rotation matrix which relates the LCS at time $t$ to the LCS at time $t+\delta t$. Then

$$
\mathbf{R}(t+\delta t)=\mathbf{R}(t) \cdot \mathbf{C}(t) .
$$

Using (2), the change rate of DCM can be expressed by

$$
\frac{d \mathbf{R}(t)}{d t}=\mathbf{R}(t) \cdot \mathbf{\Omega} .
$$

For a short time period $[t, t+\delta t]$, the solution to (4) is

$$
\mathbf{R}(t+\delta t)=\mathbf{R}(t) \cdot \exp \left(\int_{t}^{t+\delta t} \mathbf{\Omega}(t) d t\right) .
$$

Letting $\varpi=\left\|\omega_{x}, \omega_{y}, \omega_{z}\right\|$, the DCM update equation is obtained as each new angular velocity sample comes

$$
\mathbf{R}(t+\delta t)=\mathbf{R}(t) \cdot\left(\mathbf{I}+\frac{\sin (\varpi \delta t)}{\varpi} \mathbf{\Omega}+\frac{1-\cos (\varpi \delta t)}{\varpi^{2}} \mathbf{\Omega}^{2}\right)
$$

\section{Position Tracking}

With the DCM updated, it becomes possible to project the acceleration vector $\mathbf{a}(t)=\left[a_{x}(t), a_{y}(t), a_{z}(t)\right]^{T}$ in the LCS into acceleration vector $\mathbf{a}^{w}(t)=\left[a_{x}^{w}(t), a_{y}^{w}(t), a_{z}^{w}(t)\right]^{T}$ in the GCS

$$
\mathbf{a}^{w}(t)=\mathbf{R}(t) \cdot \mathbf{a}(t) .
$$

With the initial global velocity $\mathbf{v}^{w}(0)$ and initial global displacement $\mathbf{s}^{w}(0)$ known respectively, the position of a pedestrian can be tracked by subtracting the acceleration of gravity and then integrating the remaining acceleration once to obtain global velocity, and twice to obtain the displacement in the GCS. Using the rectangular rule, the integration can be implemented to the samples arriving from the accelerometer as

$$
\begin{aligned}
& \mathbf{v}^{w}(t+\delta t)=\mathbf{v}^{w}(t)+\left(\mathbf{a}^{w}(t+\delta t)-\mathbf{g}^{w}\right) \cdot \delta t \\
& \mathbf{s}^{w}(t+\delta t)=\mathbf{s}^{w}(t)+\mathbf{v}^{w}(t+\delta t) \cdot \delta t .
\end{aligned}
$$

\section{INTEGRAL DRIFT REDUCTION}

Theoretically, the position of a pedestrian can be tracked through double integration of acceleration in the GCS after the acceleration of gravity component is filtered out. However, low-cost sensors are susceptible to drift errors. The integral drift caused mostly by the biases perturbing both angular velocity and acceleration will result in error in positioning that grows

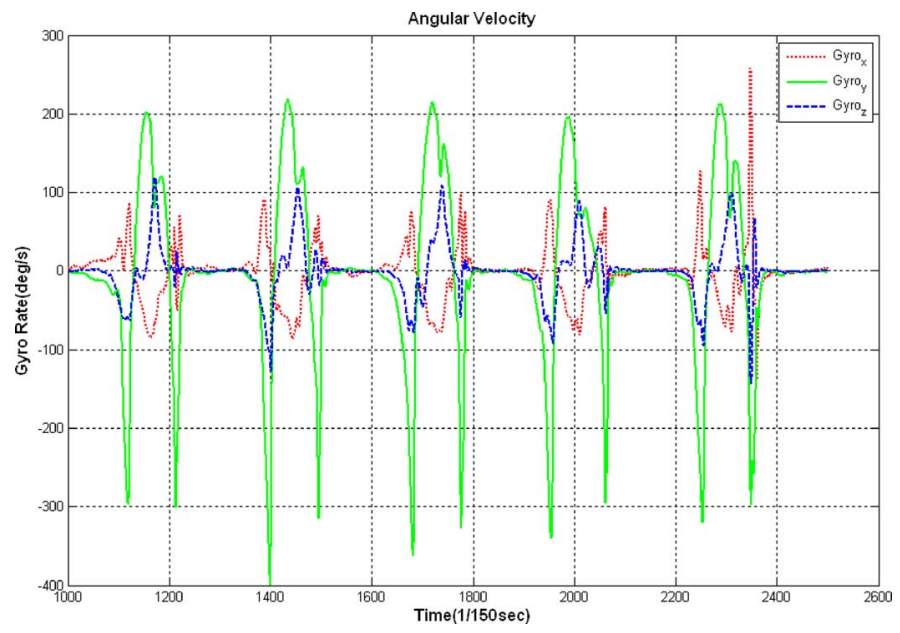

Fig. 3. Measured angular velocity.

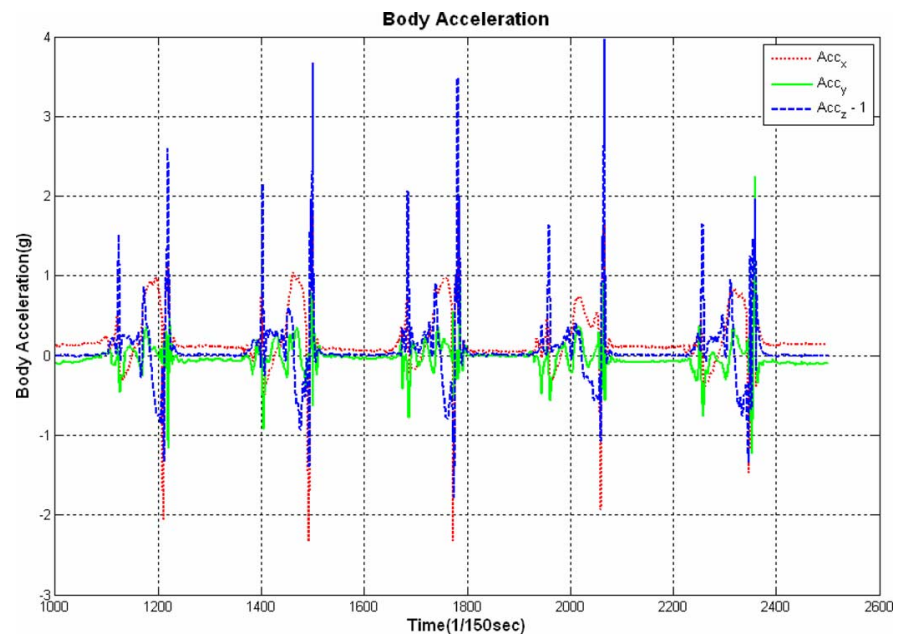

Fig. 4. Measured acceleration.

quadratically with time. Moreover, a small tilt error will also cause positioning error in the horizontal plane within the GCS. This tilt error can be treated as a certain type of acceleration bias. Therefore, integral drift correction is essential for position tracking, and solutions have to be found. This section introduces the drift reduction methods, including some novel ones we are proposing.

\section{A. Stance Phase Detection}

The human motion during a walk is cyclical and significantly consistent among individuals. During each stride, the horizontal velocity of a foot repeatedly changes from stationary, accelerating, decelerating, and to stationary again. Each stride can be divided into two phases: the stance phase and the swing phase. During the stance phase, the foot, or part of the foot, is placed on the ground. If this phase can be detected reliably from sensor data, the velocity can be reset every stride, hence reduce drift accumulation, which would otherwise trouble the data processing in the following strides. Theoretically, in order to detect stance phase we can use sensor data, including all three components of angular velocity, acceleration, magnetic field in the LCS, as shown in Figs. 3-5, respectively. In these figures, the 


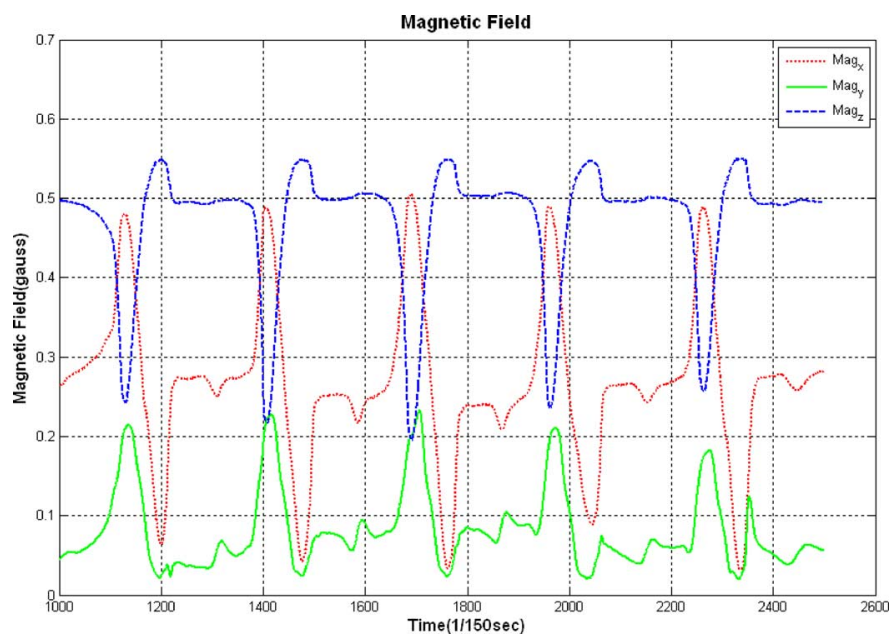

Fig. 5. Measured magnetic field.

stance phases are the periods when the signal waveforms are almost stationary while the swing phases are the periods when the waveforms are very unsteady.

It can be observed that the $y$ axis angular velocity and the $z$ axis acceleration are the most significant indicators of stride events. However, due to unsteady tilts of the sensor module, in a stance phase the components of measured acceleration on horizontal plane and vertical direction are not exactly 0 and $g$, respectively. This disables the acceleration as a candidate for detecting stance phases. In this paper, the $y$ axis angular velocity is selected to detect the stance phases. This method is novel compared with those already used [4], [5], [7].

The $y$ axis component of angular velocity is chosen because its amplitude is not only near zero in stance phases but also the largest among all the components of angular velocity in swing phases. So an empirical threshold of amplitude is found and used to distinguish stance phases. However, during swing phases there are some zero-crossings, which may be mistakenly judged as stance phases. Hence, another threshold on time period should be used to prevent such mistakes. If the angular velocity is within the threshold for a certain period of time, the stride is confirmed to be in a stance phase. Otherwise, the stride is in a swing phase.

\section{B. Quasi-Static Orientation Calculation}

In order to determine the orientation of the sensor module for rotation representation and transformation between the GCS and the LCS, the Euler angles must be determined. In swing phases, the Euler angles are calculated through integration of the angular velocity over time assuming that the original orientation of the sensor module is known. However, the original orientation is usually unknown in reality. In addition, the gyroscope signal summed during integration is perturbed by gyroscope bias. Thus the accuracy of the orientation will decay with time. Therefore, magnetometers, which can detect the magnetic field of the earth, must be used with gyroscopes and accelerometers to find the orientation in the beginning of a walk and to update the orientation in each stance phase, when the sensor module is in a quasi-static status.

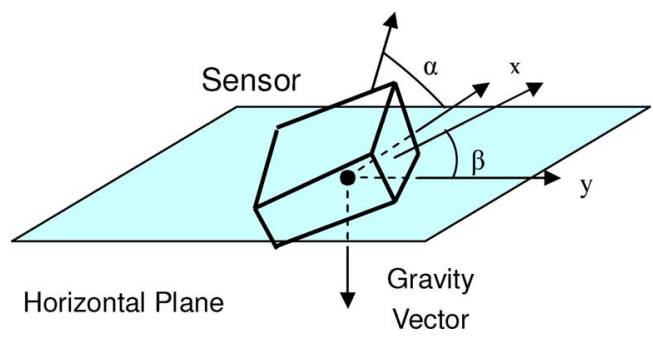

Fig. 6. $x$-tilt and $y$-tilt assignments relative to ground.

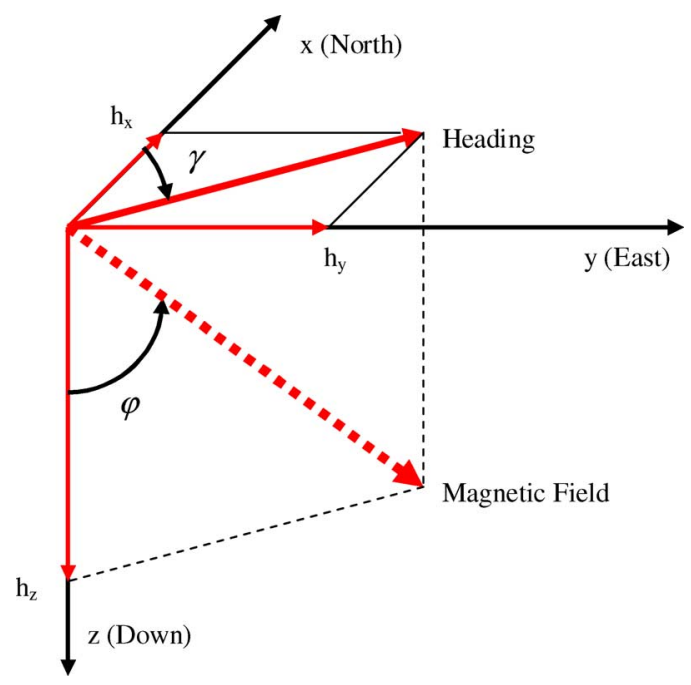

Fig. 7. Heading defined in the horizontal $x-y$ plane.

To find the quasi-static orientation, the Euler angles $\varnothing$, è, and ö must be determined. Because gyroscopes cannot be used in a quasi-static status, signals from accelerometers and magnetometers have to be used to calculate the orientations of the sensor module. However, these angles cannot be obtained directly. To resolve this problem, the tilts and heading of the sensor module need to be investigated.

Both $x$-tilt $\alpha$ and $y$-tilt $\beta$ of the sensor module, or the sensor on it, as shown in Fig. 6, can be found simultaneously using acceleration outputs of all three axes as

$$
\begin{aligned}
& \alpha=\operatorname{atan} 2\left(a_{x}, \sqrt{a_{y}^{2}+a_{z}^{2}}\right) \\
& \beta=\operatorname{atan} 2\left(a_{y}, \sqrt{a_{x}^{2}+a_{z}^{2}}\right)
\end{aligned}
$$

where the two-argument function atan 2 is a variation of the arctangent function. For any real arguments $x$ and $y$ not both equal to zero, $\operatorname{atan} 2(y, x)$ is the angle in radians between the positive $x$ axis of a plane and the point given by the coordinates $(x, y)$ on it.

The heading of a pedestrian, or the sensor module on it, can be calculated using the $x$ axis and $y$ axis magnetic field outputs if the sensor module is level in a horizontal plane. As shown in Fig. 7, only the $x$ and $y$ components of the earth's magnetic field are used when determining the heading, or the direction of sensor module. The heading $\gamma$ is defined by

$$
\gamma=\operatorname{atan} 2\left(h_{y}, h_{x}\right)
$$


No matter whether a person is standing or walking, the sensor module attached to the human body is not confined to a flat and level plane. This makes it more difficult to determine the heading direction, because the tilt of the $x$ axis and that of the $y$ axis are always changing and the sensor module cannot stay horizontal to the earth's surface. Errors caused by tilt angles can be large if not compensated.

To compensate the tilts of the sensor module when it is in an arbitrary position, the LCS is transformed into the GCS through three rotations with Euler angles $\psi, \theta$, and $\phi$ in sequence. These Euler angles are related to the tilts but they are not all the tilts of the sensor module. Thus, a new algorithm has to be established. The acceleration of gravity is to be used in this algorithm. But unfortunately, the acceleration signals acquired from the sensor module contain both the acceleration of the gravity and that caused by human motion. In order to remove the later while not to disturb the phase of the measured signals, a line-phase finite impulse response (FIR) low-pass filter (LPF) is used. The cutoff frequency of the filter is less than $1 \mathrm{~Hz}$ because it typically takes 1 second for a person to complete a stride. The filtered acceleration is then normalized and redefined as a gravity vector $\mathbf{g}^{b}$ in LCS.

Rotations of the gravity vector from the GCS to the LCS are given by

$$
\mathbf{g}^{b}=\mathbf{R}^{T} \cdot \mathbf{g}^{w}=[-\sin \theta, \sin \phi \cos \theta, \cos \phi \cos \theta]^{T}
$$

where $\mathbf{g}^{b}=\left[g_{x}, g_{y}, g_{z}\right]^{T}$, and $\mathbf{g}^{w}=[0,0,1]^{T}$. Therefore

$$
\theta=\operatorname{atan} 2\left(g x, \sqrt{g y^{2}+g z^{2}}\right)
$$

which is actually the same result as in (10) when the sensor module is not moving. Using (13) and (14), we obtain

$$
\phi=\operatorname{atan} 2\left(g_{y} \operatorname{sign}(\cos \theta), g_{z} \operatorname{sign}(\cos \theta)\right)
$$

which is different compared with $\beta$ in (11). Hence, the $\phi$ angle of roll is not the $y$-tilt angle $\beta$. $y$-tilt should not be used as roll for orientation calculation and magnetometer compensation.

After both pitch $\theta$ and roll $\phi$ are found from acceleration data, the attitude, or yaw $\psi$, can be found from magnetic field data by solving the following simultaneous equation:

$$
\mathbf{m}^{b}=\mathbf{R}^{T} \cdot \mathbf{m}^{w}
$$

where $\mathbf{m}^{b}=\left[m_{x}, m_{y}, m_{z}\right]^{T}, \mathbf{m}^{w}=[a, 0, b]^{T} a$ and $b$ are the horizontal and vertical component of the magnetic field of the earth vector, respectively) and $\mathbf{R}^{T}=\mathbf{R}_{1}^{T}(\psi, z) \cdot \mathbf{R}_{2}^{T}(\theta, y)$. $\mathbf{R}_{3}^{T}(\phi, x)$. Hence, we get

$$
\mathbf{R}_{1}^{T}(\psi, z) \cdot \mathbf{m}^{w}=\mathbf{R}_{2}^{T}(\theta, y) \cdot \mathbf{R}_{3}^{T}(\phi, x) \cdot \mathbf{m}^{b} .
$$

If $h_{x}, h_{y}$, and $h_{z}$ are the projections of magnetic field on the horizontal plane and the $z$ axis (pointing downward), respectively, i.e., $\mathbf{h}=\left[h_{x}, h_{y}, h_{z}\right]^{T}=[a \cos \psi,-a \sin \psi, b]^{T}$. Then, the compensated magnetic field can be obtained as

$$
\begin{aligned}
h_{x} & =m_{x} \cos \theta+m_{y} \sin \theta \sin \phi+m_{z} \sin \theta \cos \phi \\
h_{y} & =m_{y} \cos \phi-m_{z} \sin \phi
\end{aligned}
$$

$$
h_{z}=m_{x} \sin \theta+m_{y} \cos \theta \sin \phi+m_{z} \cos \theta \cos \phi .
$$

Accordingly, the heading on the horizontal plane, or the Euler angle yaw, can be defined as

$$
\psi=\operatorname{atan} 2\left[-h_{y}, h_{x}\right] .
$$

The angle $\psi$ calculated in (21) is actually a compass reading, i.e., the heading relative to magnetic north. To get the true heading, denoted as $\psi_{T}$ here, the magnetic declination $D$ must be deduced from the compass reading $\psi$

$$
\psi_{T}=\psi-D
$$

where $D$ is the difference between magnetic north and true north. This difference is caused by the tilt of the earth's magnetic field generator relative to the earth's spin axis.

\section{Stance Phase Updates}

Only after the quasi-static orientation is calculated, the inertial tracking introduced in Section II becomes practical. Moreover, stance phase updates (SPU), the updates of velocity and orientation during stance phases, and swing phase drift corrections (SPDC), the corrections to integral drift during swing phases, can be implemented to increase the accuracy of pedestrian tracking. In this paper, SPUs include both zero-velocity updates and heading updates.

Zero-velocity updates have actually been mentioned in Section I implicitly as the essence of the synergy of INS and PDR. Theoretically, the velocity in the GCS is zero during the stance phase. In practice, zero-velocity updates are performed when gyroscope signals drop below a certain threshold over a certain period that can be empirically determined. The purpose of these updates is mainly to ensure no movement measured during the stance phases thus limits the integral drift.

Heading updates are proposed in this paper as a novel method to solve the problem of integral drift caused by gyroscope bias. Because of the bias of the gyroscope itself, the integral drift of rotated angles grow linear with time. To guarantee this drift under control, the rotated angles, which are the result of integration to 3-D angular velocity over time, should also be updated with data from other than gyroscopes. In this paper, the rotated angles in stance phases are updated by the yaw, pitch and roll angles calculated from acceleration data and magnetic field data, but not from the integration of angular velocity over time.

\section{Swing Phase Drift Correction}

In order to obtain a higher accuracy of position tracking, SPUs, including zero-velocity updates and heading updates during stance phases, are not enough. A novel method to correct drift must be incorporated during the swing phase of each stride. Unfortunately, the methods used in stance phases can not be applied in swing phases because there is nothing stationary in these phases.

However, the global velocity, i.e., the velocity in the GCS, should be zero in both the beginning and the end of a swing phase. In most cases, even though the global velocity is set to zero with zero-velocity update in the beginning of each swing 
phase, the velocity in the end of the swing phase is not zero. We assume that this is caused by a certain constant "bias" of the global acceleration. This "bias" is actually resulted from the combination of drifts from both accelerometers and gyroscopes. This bias can be estimated from the difference of the velocity in the beginning and the velocity at the end of a swing phase. Consequently, both the global velocity and the global displacement during a swing phase have to be recalculated with the estimated bias to be removed from the calculated global acceleration.

On the other hand, the angles tracked by gyroscopes are different with those measured by magnetometers in the end of each swing phase. Because the angles tracked by gyroscope are obtained from summing the angular velocity over time, they are susceptible to integral drift and contribute mostly to angular errors. Similarly to the global velocity, we assume the difference is caused by constant "biases" of the gyroscopes and then estimate angles from this difference. Finally the estimated "biases" are removed from angular velocity in the recalculation of DCM.

\section{E. Calibration}

Further improvement on the accuracy of positioning can be achieved by removing the actual biases of accelerometers, magnetometers and gyroscopes, respectively.

The method to estimate constant bias error of a gyroscope is to take a long term average of the gyroscope's output while it remains still. Once the bias is obtained, it is important to subtract the bias from the output of the gyroscope.

Theoretically, the bias of an accelerometer can be determined by measuring the long term average of its output when it is still. In practice, this is complicated by the gravity, because a component of gravity applied to the accelerometer will appear as a bias. Therefore, in order to measure the bias, we have to know the exact orientation of the device with respect to the gravitational field.

The calibration of a magnetometer is important because the output of the magnetometer is used to correct the integral drift of a gyroscope. However, it is sophisticated too. In reality, the effect of ferrous materials nearby changes the altitude measured by magnetometer. This magnetic declination is known as the hard iron distortion. In addition, the earth's magnetic field and magnetically soft material surrounding the magnetometer affect each other and result in soft iron distortion.

To compensate for the hard iron distortion, the sensor module is rotated on a horizontal plane. An irregular circle, or actually an ellipse, can be formed from the horizontal components of all the readouts of magnetometer as shown in Fig. 8. The offsets caused by the hard iron distortion can be determined from the circle and compensated by subtracting it from magnetometer signals. The removal of soft iron distortion needs more calculation than a simple subtraction. One way to remove this distortion is to rotate the reading by $45^{\circ}$, scale the major axis to change the ellipse to a circle, and then finally rotate the reading back by the same angle. This will result in the desired circular output response shown in Fig. 9. The selection of rotated angle is based on experience from repeating experiments and comparison with different methods.

After both hard iron distortion and soft iron distortion have been compensated, bias of the magnetometer itself is calculated

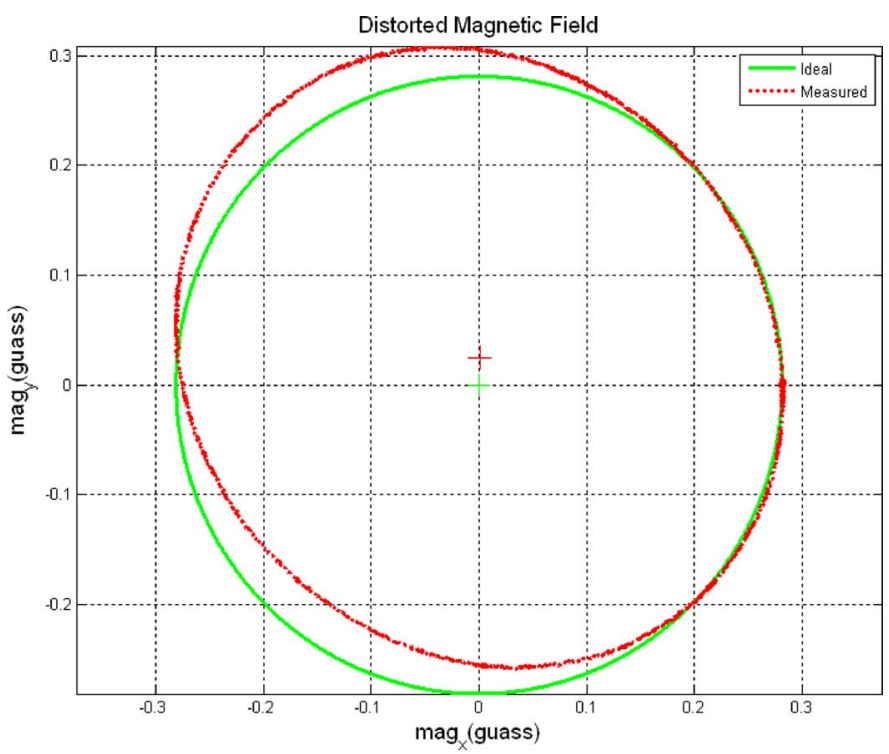

Fig. 8. Output $(x, y)$ of magnetometer rotated horizontally in the earth's field with both hard iron distortion and soft iron distortion.

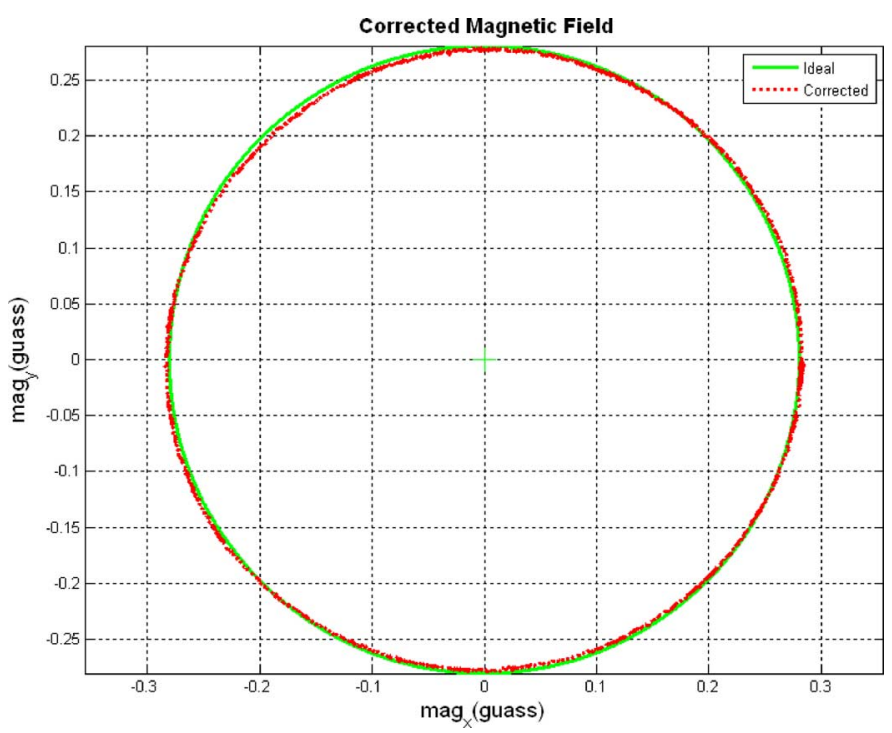

Fig. 9. Output $(x, y)$ of magnetometer rotated horizontally in the earth's field with both hard iron distortion and soft iron distortion compensated.

when both the precise orientation of the sensor module with respect to the magnetic north and those with respect to the gravitational field are known.

\section{Field TESTING AND RESUlts}

This section focuses on the implementation of the methods discussed, developed and proposed in the previous sections, and presents the results of experimental study. First, a sensor module is carefully selected. Then, the mounting of the sensor module is introduced. After that, tracking of a pedestrian walking along a square path is demonstrated step by step. Finally, the results of more field experiments are presented.

\section{A. Sensor Module Selection}

Many factors, including size, cost, dynamic range, sampling rate and bias, have to be considered in choosing an appropriate 


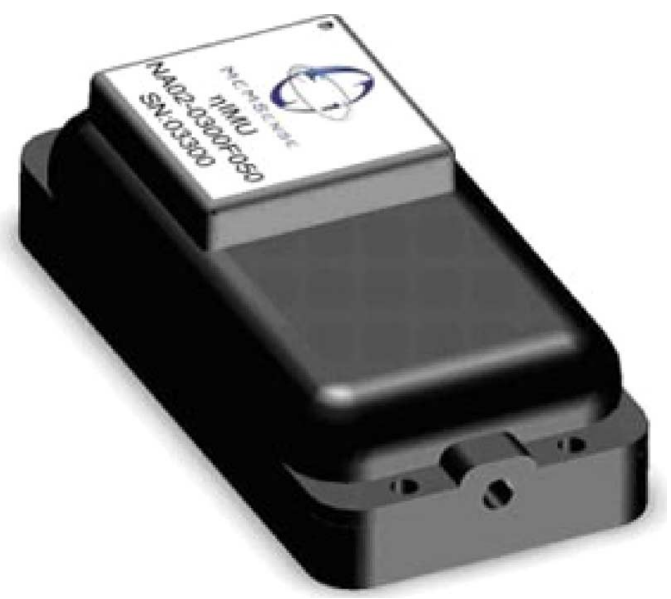

Fig. 10. Sensor module selected

sensor. First, the size of the sensor module should be small enough to be installed unobtrusively on a footwear. Consequently, all 3-D gyroscopes, 3-D accelerometers, and 3-D magnetometers should be integrated into, actually orthogonally mounted in, this inertial/magnetic sensor module. Second, the price of this sensor module should be low enough to meet budget constraints for the targeted applications. Third, the dynamic range of the sensor module should be appropriate. From Figs. 3 and 4, the sensor module may experience maximum angular velocity of $500^{\circ} / \mathrm{s}$ and maximum acceleration of $5 \mathrm{~g}$ 's or even higher. Fourth, the sampling rate should be high enough to avoid sampling errors. Considering the availability of sensor modules in the market, we assume the sampling rate of the sensors should be at least $100 \mathrm{~Hz}$. Finally, because of the sensitivity of sensors to temperature, each sensor in a module should be individually compensated for bias, which is a function of temperature.

With all these considerations a nano inertial measurement unit (nIMU) was selected to build the prototype for testing. The nIMU, as shown in Fig. 10, provides serial digital outputs of 3-D acceleration, 3-D angular velocity, and 3-D magnetic field [14].

\section{B. Sensor Module Mounting}

In the experiments related to this paper, the sensor module is mounted on the arch, i.e., the upper of closed footwear, and fixed by laces or some specific vamp itself, as shown in Fig. 11. No matter how the sensor module is installed, it should be guaranteed that there is the least relative movement between the sensor module and the foot-ware.

Moreover, an extended data cable runs up the pant leg. A battery pack is fixed with belts or even simply be placed in a pocket. The laptop computer has to be hold with both hands. While walking, the operation of the data logging is monitored in case of any problems.

\section{Tracking on a Square Path}

Fig. 12 shows original acceleration, gyroscope and magnetic field signals measured from sensors respectively. In the upper part of the figure, after gravity $(1 \mathrm{~g})$ is deducted, the $z$ axis local

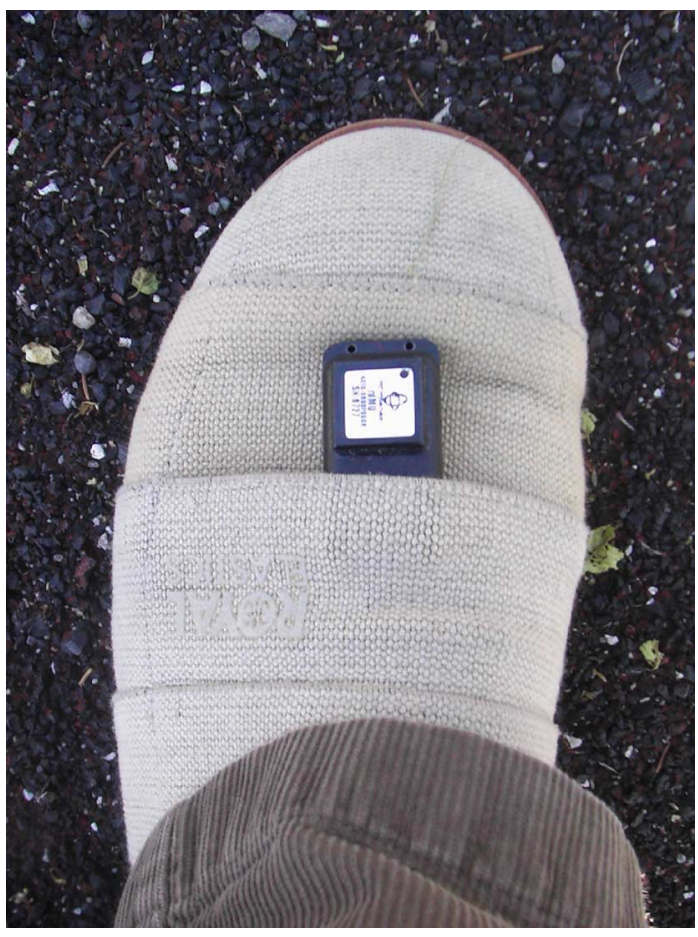

Fig. 11. Sensor module mounted on a shoe.
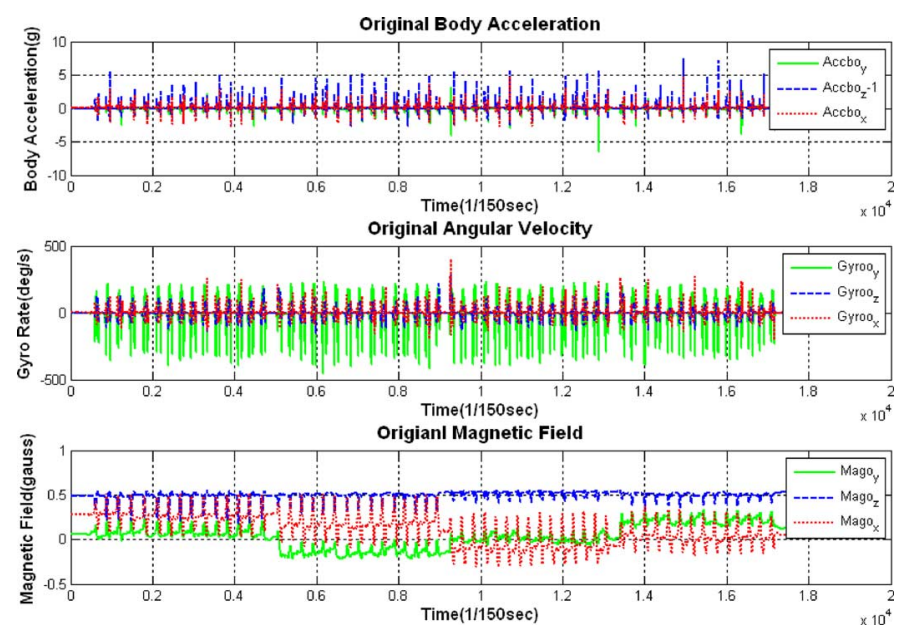

Fig. 12. Original sensor signals.

acceleration still has a larger amplitude than that of either $x$ axis or $y$ axis acceleration. Due to existing tilts of the sensor module, the average acceleration actually is not zero at stance phase. These tiny offsets from zero are components of gravity itself. In the middle part of the figure, the $y$ axis angular velocity is significantly larger than the others during the swing phase. During the stance phase, the angular velocity of each axis is near zero. In the bottom of this figure, the magnetic field signals goes to their peaks in the swing phase, changes their average value when the walking direction is turned. It is interesting to notice that the $z$ axis component is relatively stable. This is because the experiment is conducted on an almost level ground and thus the component on the $z$ axis does not change a lot.

The upper part of Fig. 13 shows quasi-static local acceleration resulting from LPF with cut-off frequency of less then $1 \mathrm{~Hz}$. 


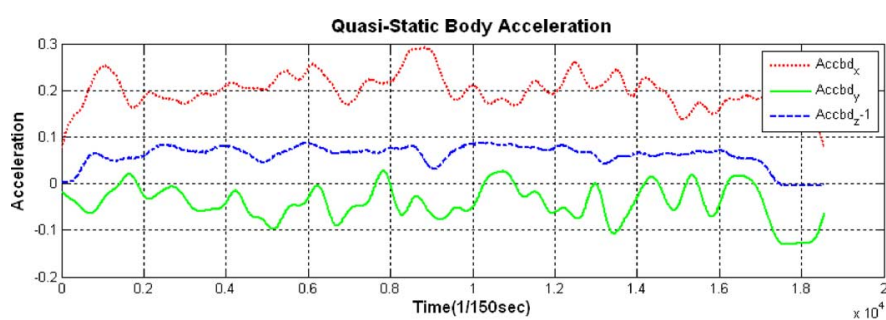

Pitch and Roll

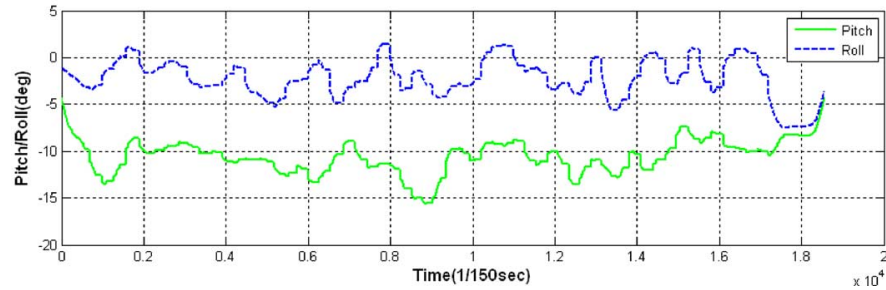

Fig. 13. Pitch and roll of the sensor module during walking.
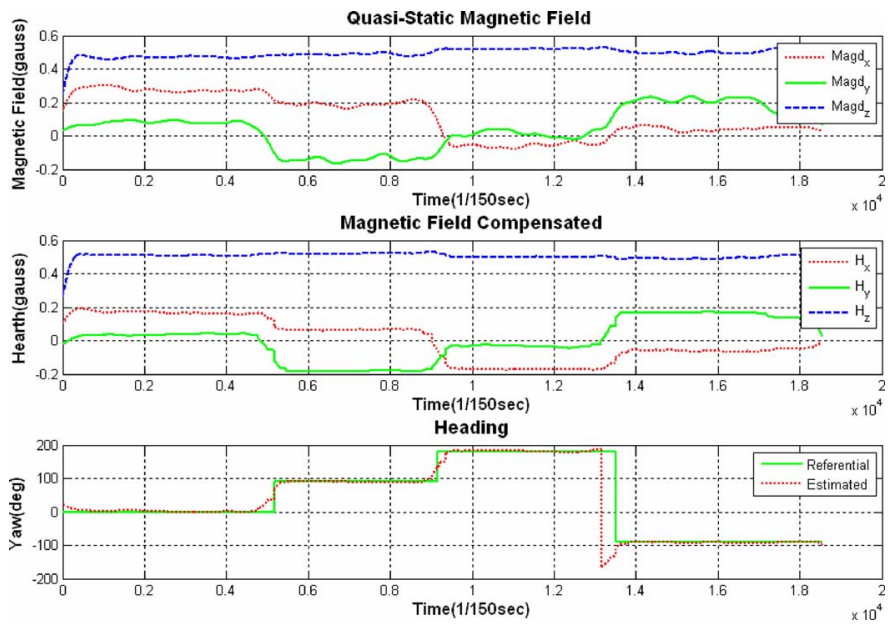

Fig. 14. Calculation of heading.

As described in Section III, these signals are used to calculate the pitch and roll of the sensor module. The result demonstrates the continuous changing of pitch and roll of the sensor module during walking. The change of pitch is mostly between $-7^{\circ}$ and $-15^{\circ}$ while the change of roll is mostly between $1^{\circ}$ and $-5^{\circ}$. The changing of roll is mostly because of an uneven ground, while the changing of pitch is caused by both the walking itself and the uneven ground.

Fig. 14 shows how the heading of the sensor module, consequently that of the pedestrian, is calculated. In the upper part of the figure, the original magnetic field signals from the magnetometer are wavy because of the changing of pitch and roll of human foot. In the middle part of the figure, after the magnetic field signals are compensated for tilts as depicted in Section III, they become much smoother. Then, these compensated signals are used to calculate the heading, or yaw, which is illustrated in the lower part of the figure. The heading demonstrates the sequence of directions of the clockwise walk on a square path: northbound, eastbound, southbound and westbound. The ideal yaw is for reference only: the turns of direction can not be that sharp during human walking.
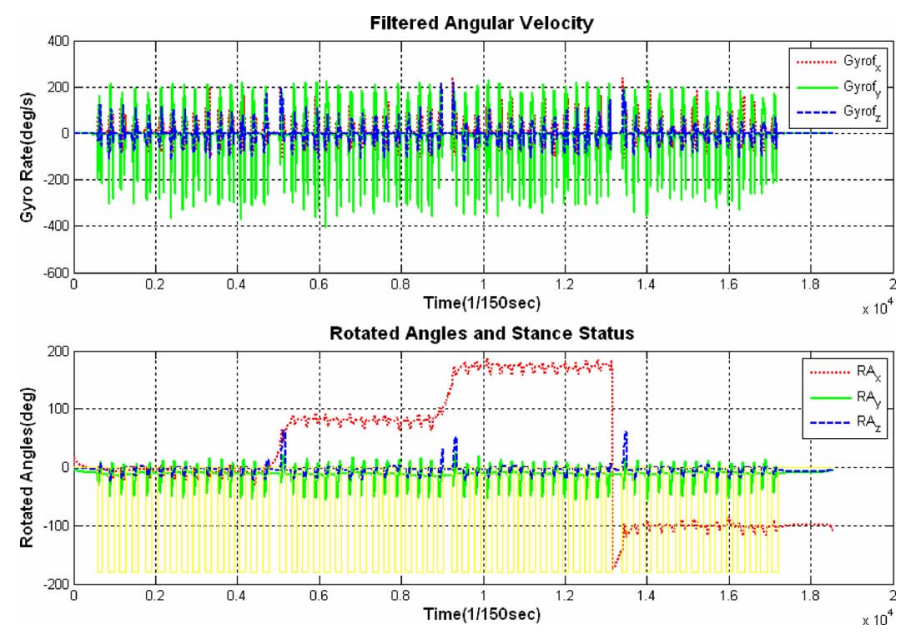

Fig. 15. Angular velocity and rotated angles.
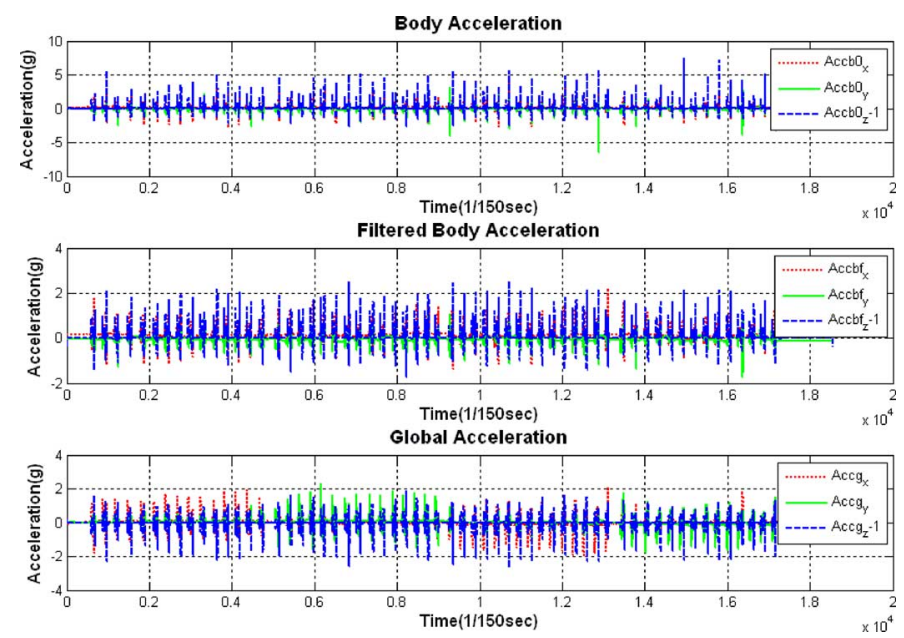

Fig. 16. Local acceleration and projected global acceleration.

The upper part of Fig. 15 depicts the angular velocities with the high-frequency noise removed by LPF. The $y$ axis angular velocity is significantly larger than the others during walking. This is because most rotations are around $y$ axis. However, when the walking direction is changed, the amplitude of angular velocity around $z$ axis also becomes large because there is a significant rotation on the horizontal level while turning. The lower part of the figure shows the rotated angles obtained from the integration to gyroscope signals over time. During a walk along a straight line, the angles do not change greatly. Only when the walking direction is changed, the angle around $z$ axis changes about $90^{\circ}$. By the way, the switching between stance phases and swing phases are also illustrated in this figure.

Fig. 16 compares the original acceleration and the global acceleration, which is the result of projection from filtered local acceleration. From the local acceleration, it is not possible to identify the direction of walk because there is no significant difference between components of acceleration. On the contrast, it is possible to estimate the walking direction from the global acceleration. For example, when walking northbound, the average of $x$ axis acceleration is positive while the average of $y$ 

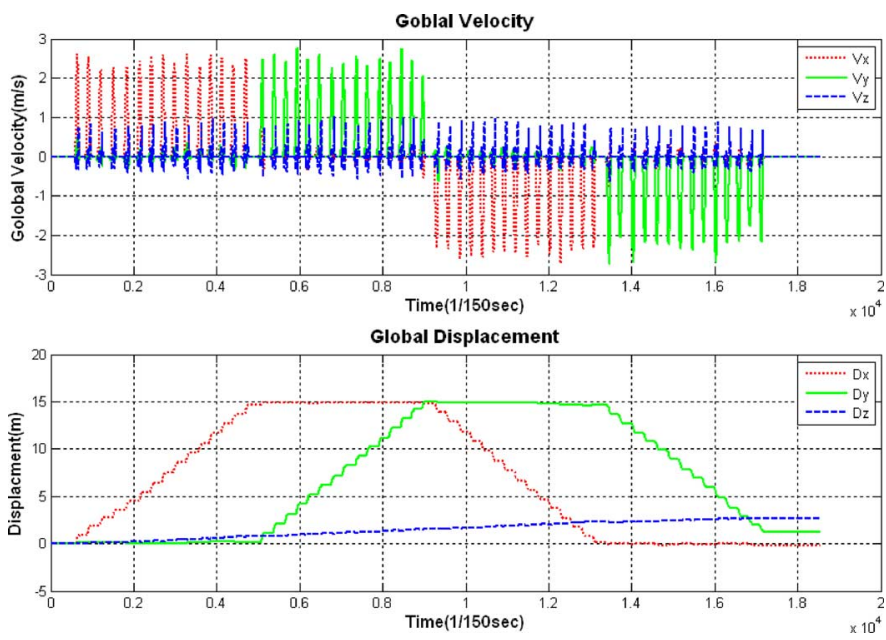

Fig. 17. Global velocities and displacements calculated.

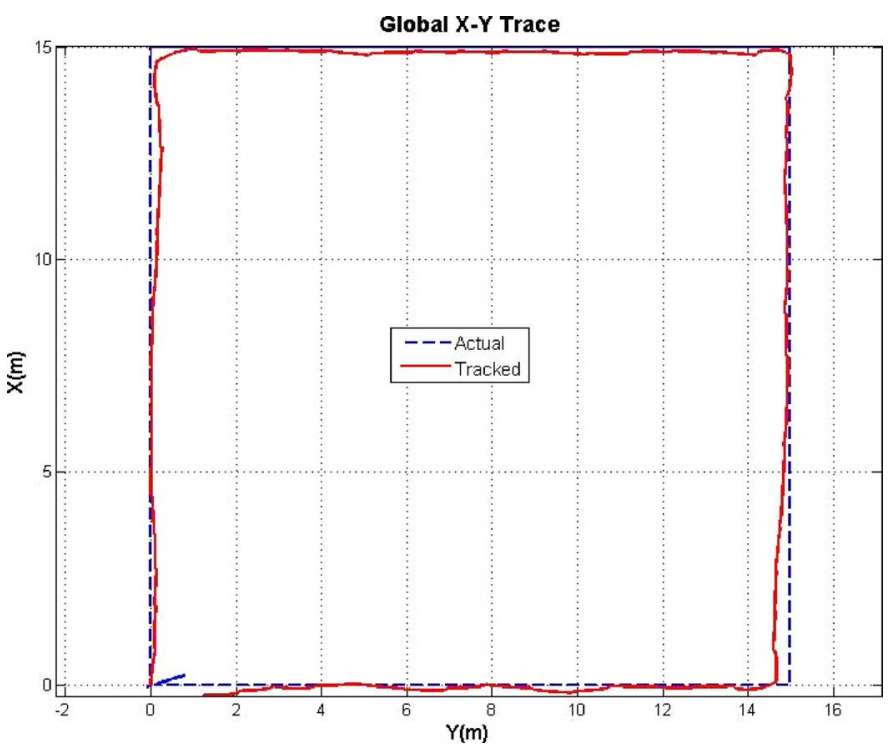

Fig. 18. Walk along a square path.

axis acceleration is around zero. It should also be noticed that the amplitude of $z$ axis acceleration is relatively stable because there is no obvious vertical movement during the field test.

The differences among acceleration signals become obvious after they are integrated once over time. As shown in Fig. 17, when walking northbound (southbound), the $x$ axis velocity is positive (negative) and its amplitude is the largest. The $y$ axis velocity is positive (negative) and its amplitude is the largest when walking eastbound (westbound). However, the amplitude of $z$ axis is almost stable. After the acceleration signals are double integrated over time, the displacements along three axes are calculated respectively. It can be observed that the displacement on a certain axis increase (or decrease) stride by stride when the walk is along that axis. It is also noticeable that there is an approximately $2.6 \mathrm{~m}$ displacement upward after a walk of 60 $\mathrm{m}$. This is mostly caused by the component of gravity on $z$ axis. Other or more sophisticated solutions have to be found for more accurate tracking vertically.

Fig. 18 shows the final result of the above experiment where the walk is along a square-shaped line. The side of the square

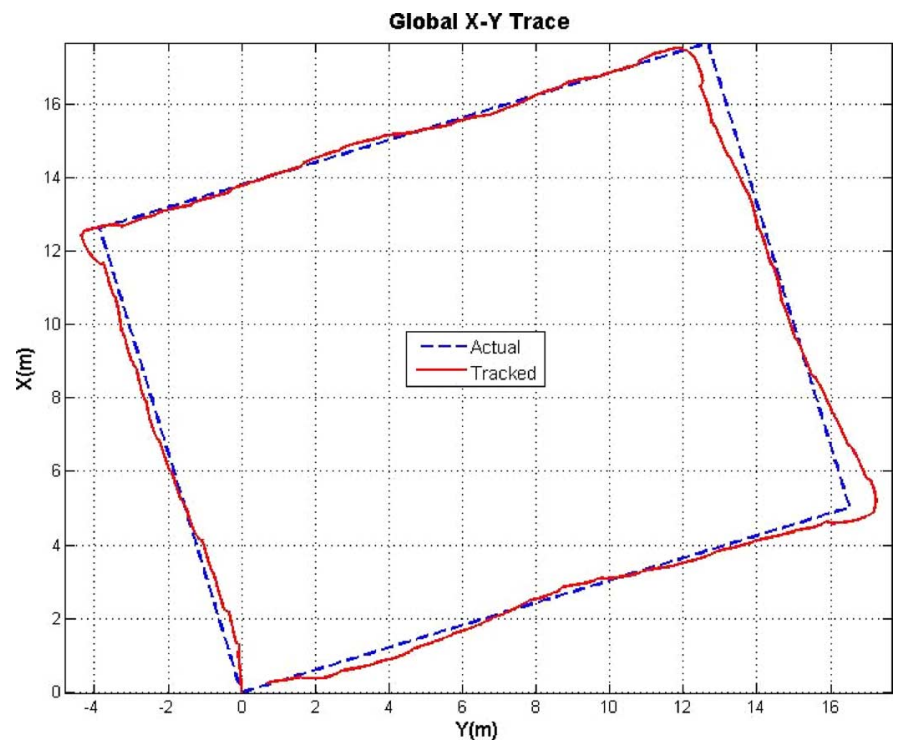

Fig. 19. Walk along a rectangle.

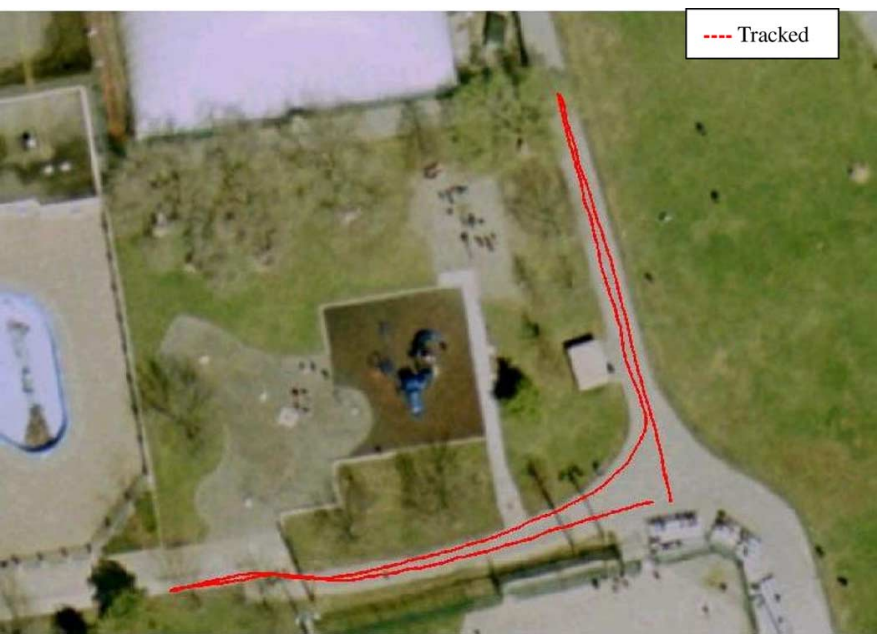

Fig. 20. Walk on a J-shaped path.

was $15 \mathrm{~m}$ in length, resulting in a total length of $60 \mathrm{~m}$. The sides were intentionally set to be either parallel or vertical to magnetic north. It is noticed that although the path is a close square loop but the estimated trace is not closed. There are about $1.2 \mathrm{~m}$ short compared with the total path of $60 \mathrm{~m}$. Hence, the accuracy of the tracking is around $2 \%$ as to the distance traveled.

\section{Tracking on Other Paths}

Fig. 19 is the final result of an experiment where a pedestrian walked along a rectangle path. The longer side of the rectangle was $17.5 \mathrm{~m}$ and the shorter side was $14 \mathrm{~m}$.

In the experiment related to Fig. 20, the walk is along a J-shaped path near a children playground. A pedestrian walked northbound for $40 \mathrm{~m}$, southbound for $36 \mathrm{~m}$, then along an arc, westbound, a little bit northbound, and then turned back eastbound in sequence. The plotted trace recorded is similar to the actual path walked.

Fig. 21 illustrates the result of tracking to a pedestrian who walked along an outdoor athletic track with total length of more 


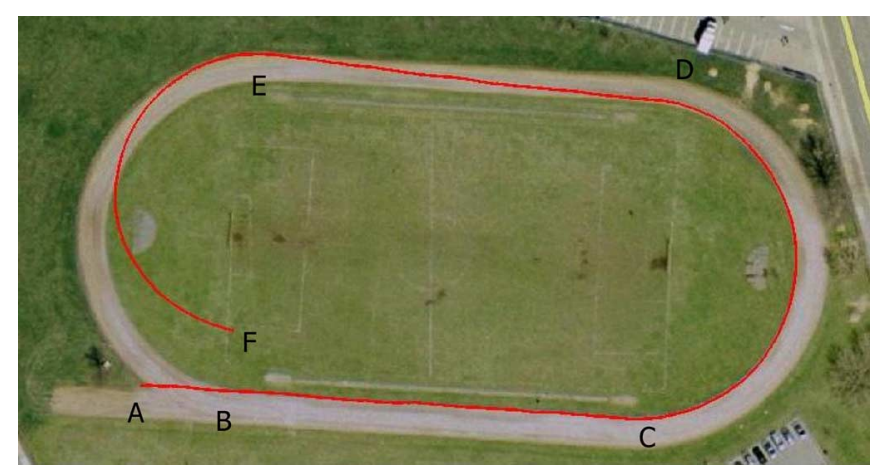

Fig. 21. Walk at a track-and-field ground.

than $400 \mathrm{~m}$. During the test, the person carrying the prototype started walking from the starting area (point A in Fig. 21) and walked along the track to point F. The tracked path is in excellent agreement with the actual path during the first $200+\mathrm{m}$ (between Point A and D). Between Point D and E, the tracking is accurate in term of distance but suffers a small error in term of orientation. This is an issue to be further investigated in future study. Between E and F, the trajectory is accurate but does not fit into the actual path due to the error caused in the path between $\mathrm{D}$ and $\mathrm{E}$. The problem between $\mathrm{D}$ and $\mathrm{E}$ is partially due to the technical reliability of the prototype, which will be improved in future study.

\section{CONCLUSIONS AND FUTURE WORK}

This paper presents a research on a pedestrian tracking system using a small-sized inertial sensor module. Based on experiments done to date, the accuracy of the system is acceptable in measuring displacement of a pedestrian. The drifted error on the horizontal plane is around $2 \%$ of distance traveled. The accuracy is also indifferent to pauses or changes in walking gaits. These results suggest that a targeted self-contained pedestrian tracking system based on MEMS sensors, instead of wireless or other technologies, can indeed be practical through synergism of INS and PDR systems and an integration of a variety of integral drift correction approaches. From the results of experiments, we can also conclude that the updates done during stance phases and the drift corrections done during swing phases are useful to limit the unbounded growth of integral drift while the algorithm to calculate the orientation of a sensor module from magnetic field data is successful.

Most important contributions of this paper are the synergism of INS and PDR technologies and the novelty in methods to reduce integral drift of tracking. These contributions improve the tracking accuracy, which is the key for the success of such a system. These novel methods include: 1) the using of $y$ axis gyroscope signal, instead of acceleration signal, to detect stance phases; 2) the calculation of heading using tilt compensated magnetic field during quasi-static periods of human walking; 3 ) the update of rotated angle, which is the result of integration to angular velocity over time, in each stance phase; and 4) the removal of "bias" of global acceleration and that of angular velocity during each swing phase.
Since the system requires no fixed infrastructure, the operational cost is proportional to the number of users, rather to the coverage area as is the case for traditional location systems. Therefore such a system can be used to enable the deployment of location-aware application, such as fire fighting operations.

For further design and development of a practical pedestrian tracking system for much wider real-life applications with higher accuracy, precision, and robustness in both indoor and outdoor environment, future work will continue with focuses on map correlation, particle filtering, integration with global navigation satellite systems (GNSS), etc.

\section{REFERENCES}

[1] J. Hightower and G. Borriello, "Location systems for ubiquitous computing," Computer, vol. 34, no. 8, pp. 57-66, Aug. 2001.

[2] H. Liu, H. Darabi, P. Banerjee, and J. Liu, "Survey of wireless indoor positioning techniques and systems," IEEE Trans. Syst., Man, Cybern. C, vol. 37, no. 6, pp. 1067-1080, Nov. 2007.

[3] R. Challamel, P. Tomé, D. Harmer, and S. Beauregard, "Performance assessment of indoor location technologies," in IEEE PLANS, Position Location and Navigation Symp. Conf. Rec., 2008, pp. 624-632.

[4] Q. Ladetto, V. Gabaglio, B. Merminod, P. Terrier, and Y. Schutz, "Human walking analysis assisted by DGPS," in Proc. GNSS, Edinburgh, U.K., May 2000.

[5] Q. Ladetto, "On foot navigation: Continuous step calibration using both complementary recursive prediction and adaptive Kalman filtering," in Proc. ION GPS, Salt Lake City, UT, Sep. 2000b.

[6] H. Leppakoski, J. Kappi, J. Syrjarinne, and J. Takala, "Error analysis of step length estimation in pedestrian dead reckoning," Inst. Navigation, pp. 1136-1142, Sep. 2002.

[7] J. Collin, O. Mezentsev, and G. Lachapelle, "Indoor positioning system using accelerometry and high accuracy heading sensors," in Proc. GPS2003, Alexandria, VA, Sep. 2003, pp. 1164-1170.

[8] Q. Ladetto and B. Merminod, An alternative approach to vision techniques: Pedestrian navigation system based on digital magnetic compass and gyroscope integration 2009. [Online]. Available: http://topo. epfl.ch/publications/sci2002.pdf

[9] O. Mezentsev, G. Lachapelle, and J. Collin, "Pedestrian dead reckoning - A solution to navigation in GPS signal degraded areas," Geomatica, vol. 59, no. 2, pp. 175-182, 2005.

[10] S. Beauregard and H. Haas, "Pedestrian dead reckoning: A basis for personal positioning," in Proc. 3rd Workshop on Positioning, Navigation and Commun., 2006, pp. 27-35.

[11] K. J. Walchko and P. A. C. Mason, "Inertial Navigation," in Proc. Florida Conf. Recent Advances in Robotics, 2002.

[12] D. H. Titterton and J. L. Weston, Strapdown Inertial Navigation Technology, 2nd ed. Reston, VA: Amer. Inst. Aeronaut. Astronaut., 2004.

[13] O. Woodman, An Introduction to Inertial Navigation, Univ. Cambridge, Cambridge, U.K., Tech. Rep. 696, 2007.

[14] nIMU Nano Inertial Measurement Unit Series Documentation2.10 ed. MEMSense, 2008.

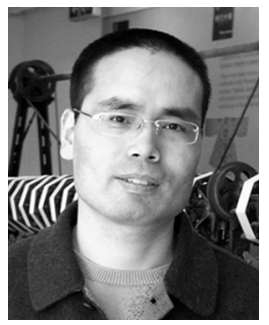

Chengliang Huang received the M.A.Sc. degree in electrical and computer engineering from Ryerson University, Toronto, ON, Canada, in 2009. He is currently working towards the Ph.D. degree at Ryerson University.

He was a Senior Engineer for eight years at Huawei Technologies Co., Ltd., and Intel Corporation. His research interests include statistical signal processing, wireless communications, system identification, and dynamic optimization. 


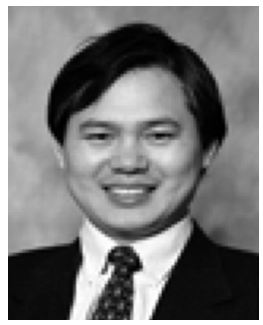

Zaiyi Liao received the Ph.D. degree in building services engineering from Hong Kong Polytechnic University in 2001 and the Ph.D. degree in engineering science from the University of Oxford, Oxford, U.K., in 2004.

$\mathrm{He}$ is currently an Associate Professor and the Director of the Master Building Science Program at Ryerson University, Toronto, ON, Canada. His current research focuses on intelligent sensors and control schemes for building automation, acoustic emission technologies for structure diagnosis, energy efficiency of residential buildings in Canada, and modeling of building systems. Dr. Liao is Professional Engineer and a member of the American Society of Heating, Refrigeration and Air-conditioning Engineers (ASHRAE).

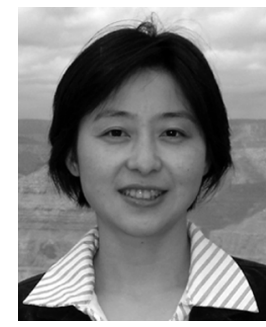

Lian Zhao received the Ph.D. degree in electrical and computer engineering from the University of Waterloo, Waterloo, ON, Canada, in 2002.

Before she joined Ryerson University, Toronto, ON, in 2003, she was a Postdoctoral Fellow with the Centre for Wireless Communications, University of Waterloo. Since 2003, she has been an Assistant Professor and then Associate Professor with the Department of Electrical and Computer Engineering, Ryerson University. She co-founded the Optic Fiber Sensing Wireless Network Laboratory in 2004. Her research interests are in the areas of wireless communications, radio resource management, power control, design and applications of the energy efficient wireless sensor networks.

Dr. Zhao is a Registered Professional Engineer in the province of Ontario, Canada. 